\title{
Anomalous origin of the left coronary artery from the pulmonary artery case report and review
}

Sandhu $\mathrm{K}^{1}$, Barron $\mathrm{D}^{2}$, Jones $\mathrm{H}^{3}$, Clift $\mathrm{P}^{4}$, Thorne $\mathrm{S}^{4}$, Butler $\mathrm{R}^{5}$

${ }^{1}$ Cardiology Specialist Registrar Royal Stoke University Hospital, University Hospitals of North Midlands.

${ }^{2}$ Consultant Congenital Heart Surgeon Birmingham Children's Hospital

${ }^{3}$ Consultant Radiologist Royal Stoke University Hospital, University Hospitals of North Midlands.

${ }^{4}$ Adult Congenital Heart Disease Queen Elizabeth Hospital Birmingham

${ }^{5}$ Consultant Interventional Cardiologist Royal Stoke University Hospital, University Hospitals of North Midlands.

All correspondence to;

Dr Kully Sandhu

Specialist Cardiology Registrar,

Royal Stoke University Hospital, University Hospitals of North Midlands.

Newcastle Road

Stoke on Trent. ST4 6QG

United Kingdom.

Email ksandhu@hotmail.com

Telephone 07888734960

Conflicts of interest; None for any of the authors for this work 


\begin{abstract}
Anomalous origin of the left coronary artery from the pulmonary artery is a rare congenital condition that proves to be fatal in most individuals during childhood due to significant left ventricular ischaemia. However there are case reports of individuals surviving into adulthood that have varying presenting symptoms. We report a case of a young male who presented to our cardiology clinic with typical ischaemic cardiac pain with no established risk factors and found to have anomalous origin of the left coronary artery from the pulmonary artery that was subsequently surgically corrected.
\end{abstract}

\title{
Key words
}

Chest pain, Coronary anomalies, coronary angiography, coronary computed tomography, cardiac surgery 


\section{Introduction}

Anomalous origin of the left coronary artery from the pulmonary artery is a rare congenital condition that often proves fatal in infants. However we present a case of a young patient presenting with angina like chest pains since childhood and subsequently underwent successful surgical correction resulting in alleviation of symptoms..

\section{Case Report}

A 25 years old normotensive, non-diabetic, non-smoker with no family history of ischaemic cardiac disease was referred to cardiology clinic by his general practitioner for progressive exertional chest pains since childhood. Since the clinical scenario did not correlate with a low risk cardiovascular profile an exercise treadmill test was performed revealing non-specific ST changes within the anterior leads after 9 minutes with accompanying chest pain. A subsequent stress echocardiogram found a dilated left ventricle (LV) of $6.1 \mathrm{~cm}$ at end diastole, normal global systolic function at rest with hypokinesia of mid and apical anterior wall during peak stress. The evidence of reversible ischaemia led to a coronary angiogram being performed via right femoral artery revealing a dominant large tortuous right coronary artery (RCA) and via collaterals to the left coronary artery (LCA) system with subsequent retrograde flow into the pulmonary artery (figure 1). An aortogram demonstrated no appreciable LCA origin. This led to a coronary computed tomography (CT) being performed that confirmed the diagnosis of anomalous origin of the left coronary artery from the pulmonary artery (ALCAPA). The large tortuous RCA originated from the aortic root, with the LCA attached to the main pulmonary artery (MPA) 
(figure 2). A curved planar reformatted CT image confirms the origin of the LCA from the MPA (figure 3).

Our patient was referred to and seen at the regional adult congenital heart disease centre.

The patient consequently underwent a translocated ALCAPA repair with bovine patch and main pulmonary artery reconstruction in May 2014. The operative findings noted a large dominant RCA with left main stem originating within the lateral aspect of main pulmonary artery $3-5$ $\mathrm{mm}$ above the post pulmonary cusp leaflet. The mitral valve was structurally normal. Mild LV systolic impairment with mitral valve insufficiency improved post operatively. Post-operative recovery was uneventful with no reported symptoms and greatly improved exercise tolerance at subsequent outpatient-follow up.

\section{Discussion}

Anomalous origin of the left coronary artery from the pulmonary artery (ALCAPA) is a rare congenital cardiovascular defect with an estimated incidence of 1 in 300000 live births, however this may be an underestimate considering patients may die prior to diagnosis. ${ }^{1}$ True incidence of older patients are not know with only case reports of patients older than 50 years of age..$^{2,3}$

Krause $^{4}$ and Brooks ${ }^{5}$ first described anomalous arteries from the pulmonary artery in 1865 and 1885 respectively from post mortem examinations. Brooks ${ }^{5}$ went on to propose that the left coronary artery had retrograde direction of blood flow to the pulmonary artery. ALCAPA may also be known as Bland, White, and Garland syndrome after a published report of the 
constellation of symptoms, electrocardiographic (ECG) changes and confirmation of ALCAPA on post mortem examination of an infant. ${ }^{6}$

Symptoms are secondary to LV ischaemia due to retrograde flow of blood from the RCA then via collaterals to LCA with draining into the pulmonary artery (PA). Therefore the foetus is protected from left ventricular ischaemia as systemic and pulmonary arterial pressures are equal intra-uterine allowing anterograde flow down both left and right coronary arteries. However during neonatal period pulmonary pressures decrease and together with ductus arteriosus closure results in an increase in systemic arterial pressure reversing the blood flow within the LCA retrograde into the PA. The severity of symptoms reflects the degree of myocardial ischaemia and is dependent on the extent of the collateralisation between RCA and LCA with greater collateralisation leading to less myocardial ischaemia resulting in fewer symptoms and increased survival. Therefore, explaining the spectrum of symptoms from death during infancy to survival into adulthood. ${ }^{7}$

Clinical presentation is often non-specific ranging from angina, syncope, arrhythmias, exertional fatigue to nocturnal dyspnoea. A review found $66 \%$ of patients had symptoms of angina, dyspnoea, palpitations, or fatigue. $17 \%$ presented with ventricular arrhythmia, syncope, or sudden death and $14 \%$ of patients were asymptomatic. ${ }^{1}$

ECGs often show lateral ischaemia or evidence of previous anterior myocardial infarction with presence of $Q$ waves in as many as $50 \%$ of patients and left ventricular hypertrophy being seen in up to $28 \%$ with $4 \%$ of patients having a normal ECGs. ${ }^{1}$ A dilated RCA with retrograde Doppler flow from LCA to PA with visualisation of septal flow due to collaterals have been described as diagnostic echocardiographic criteria for ALCAPA. ${ }^{8}$ However normal echocardiograms may be 
seen in $10 \%$ of patients and the presence of ischaemia in stress ECG or imaging such as in our patient is well documented. ${ }^{1}$

Coronary angiography alludes to the diagnosis with the presence of a large tortuous RCA with collaterals filling the LCA system as seen with our patient, figure 1. Non-invasive imaging modalities such as cardiac CT and or MRI allow visualization of the origin of the LCA from the PA, dilated tortuous RCA and collateral arteries with CMR having the advantage of being able to assess myocardial viability and ischaemia. ${ }^{9}$ Figures 2 and 3 show CT images of our patient confirming the diagnosis of ALCAPA.

Surgical correction is the treatment of choice in suitable patients and been shown to improve chronic cardiac ischaemia with encouraging post-operative long term survival in infants undergoing surgical ALCAPA correction of $94.8 \%{ }^{10}$ and $92 \%{ }^{11}$ with only handful of case reports reporting survival after ALCAPA surgical repair in adults. On both 3 and 6 month follow up our patient reported no symptoms with greatly improved exercise tolerance.

\section{Conclusion}

ALCAPA is a rare condition. High index of suspicion required. Coronary angiography may allude to the diagnosis however cardiac CT or MRI is a requisite for diagnostic confirmation. Surgical correction is the treatment of choice, with a frank discussion of risk and benefits being considered on an individual patient basis. Long-term studies are limited but suggest good outcome. 


\section{Figures}

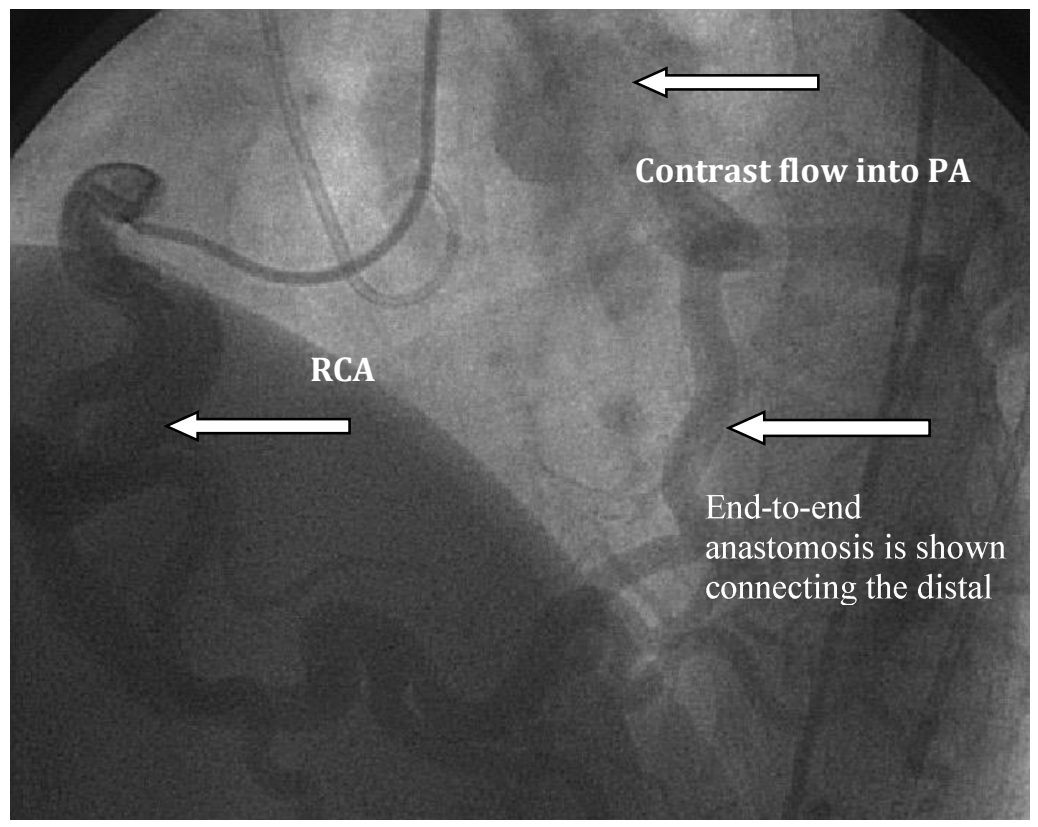

Fig. 1, Diagnostic coronary angiogram via right femoral artery illustrating the presence of a large tortuous right coronary artery (RCA) with collaterals filling the left coronary arterial system (LCA) and retrograde flow of contrast within the main pulmonary artery (PA)

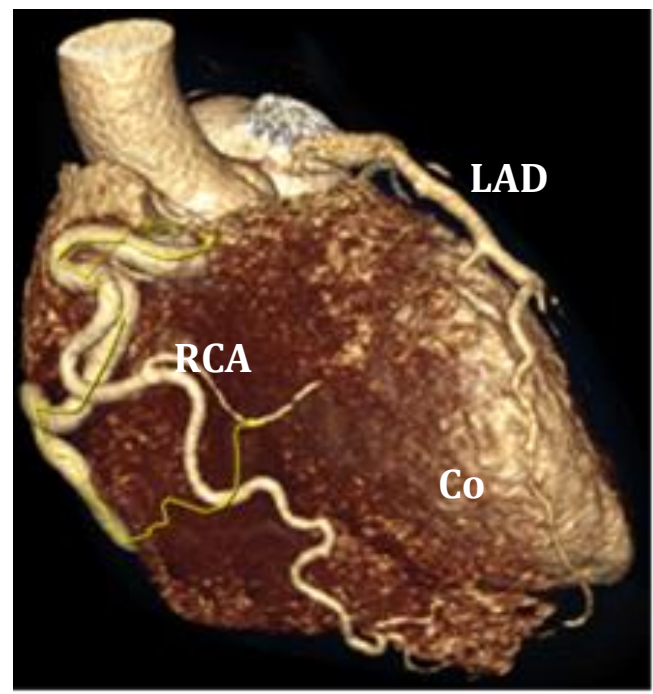

Fig. 2, Computed tomographic scan illustrating a large tortuous right coronary artery (RCA) arising from the aorta (Ao) and the left anterior descending coronary artery (LAD) attaches to the main pulmonary artery (MPA).

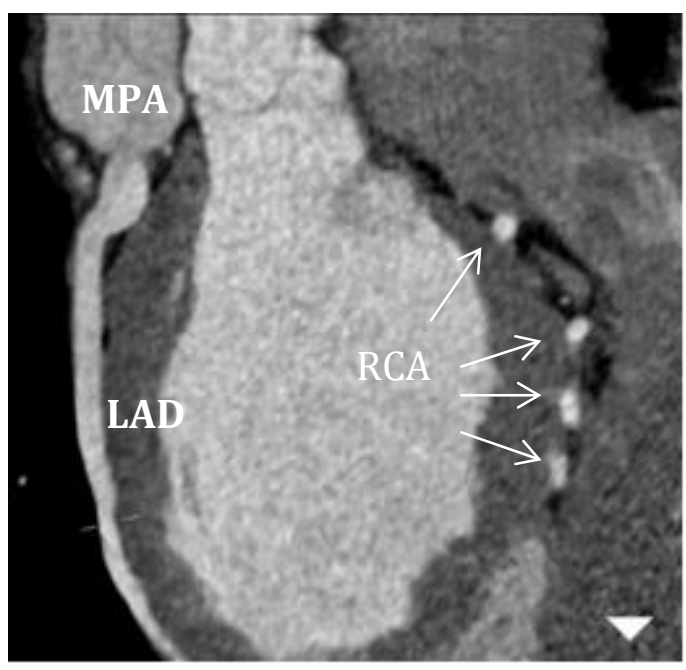

Fig. 3, Computed tomographic reformatted curved planar image showing origin of the left anterior descending coronary artery (LAD) from the main pulmonary (MPA). White arrows identify cross sections of larger tortuous right coronary artery (RCA). 


\section{References}

1. Yau JM, Singh R, Halpem EJ, Fischman D. Anomalous origin of the left coronary artery from the pulmonary artery in adults: a comprehensive review of 151 adult cases and a new diagnosis in a 53-year-old woman. Clin Cardiol, 2011.;34(4): 204-10.

2. Separham A, Aliakbarzadeh P. Anomalous left coronary artery from the pulmonary artery presenting with aborted sudden death in an octogenarian: a case report. J Med Case Rep, 2012(6): 12.

3. Fierens C, Budts W, Denef B, Van De Werf F. A 72 year old woman with ALCAPA. Heart, 2000. 83(1):E2.

4. Krause W. Uber den Ursprung einer akzessorischen A. coronaria aus der A. pulmonalis. Z Rat Med., 1865;(24): 225-229.

5. Brooks HS. Two cases of an abnormal coronary artery of the heart arising from the pulmonary artery: with some remarks upon the effect of this anomaly in producing cirsoid dilatation of the vessels. J Anat Physiol, 1885; 20(1):26-29.

6. Bland EF, White PD, Garland J. Congenital anomalies of the coronary arteries: report of an unusual case associated with cardiac hypertrophy. Am Heart J, 1933; 8:787-801.

7. Shivalkar B, Borgers M, Daenen W, Gewillig M, Flameng W. ALCAPA syndrome: an example of chronic myocardial hypoperfusion? J Am Coll Cardiol,1994;23(3):772-8.

8. Yang YL, Nanda NC, Wang XF, Xie MX, Lu Q, He L, Lu XF. Echocardiographic diagnosis of anomalous origin of the left coronary artery from the pulmonary artery. Echocardiography, 2007;24(4):405-11.

9. Pena E, Nguyen ET, Merchant N, Dennis G. ALCAPA syndrome: not just a pediatric disease. Radiographics, 2009;29: p. 553-65.

10. Lange R, Vogt M, Horer J, Cleuziou J, Menzel A, Holper K, et al. Long-term results of repair of anomalous origin of the left coronary artery from the pulmonary artery. Ann Thorac Surg, 2007;83(4):1463-71.

11. Azakie A, Russell JL, McCrindle BW, Van Andell GS, Benson LN, Coles JG, Williams WG. Anatomic repair of anomalous left coronary artery from the pulmonary artery by aortic reimplantation: early survival, patterns of ventricular recovery and late outcome. Ann Thorac Surg, 2003;75(5):1535-41. 
